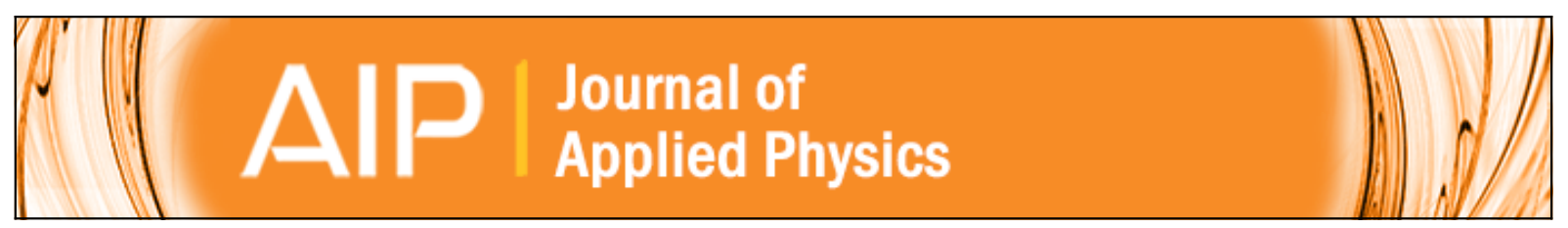

\title{
Ultraviolet laser patterning of porous silicon
}

Fidel Vega, Ramón J. Peláez, Timo Kuhn, Carmen N. Afonso, Gonzalo Recio-Sánchez, and Raúl J. Martín-

Palma

Citation: Journal of Applied Physics 115, 184902 (2014); doi: 10.1063/1.4875378

View online: http://dx.doi.org/10.1063/1.4875378

View Table of Contents: http://scitation.aip.org/content/aip/journal/jap/115/18?ver=pdfcov

Published by the AIP Publishing

\section{Articles you may be interested in}

Dynamics of fast pattern formation in porous silicon by laser interference

Appl. Phys. Lett. 105, 161911 (2014); 10.1063/1.4900431

Synthesis, properties, and applications of silicon nanocrystals

J. Vac. Sci. Technol. B 31, 020801 (2013); 10.1116/1.4794789

The role of asymmetric excitation in self-organized nanostructure formation upon femtosecond laser ablation AIP Conf. Proc. 1464, 428 (2012); 10.1063/1.4739897

Colored porous silicon as support for plasmonic nanoparticles

J. Appl. Phys. 111, 084302 (2012); 10.1063/1.3703469

Formation and post-deposition compression of smooth and processable silicon thin films from nanoparticle suspensions

J. Appl. Phys. 111, 064316 (2012); 10.1063/1.3697980

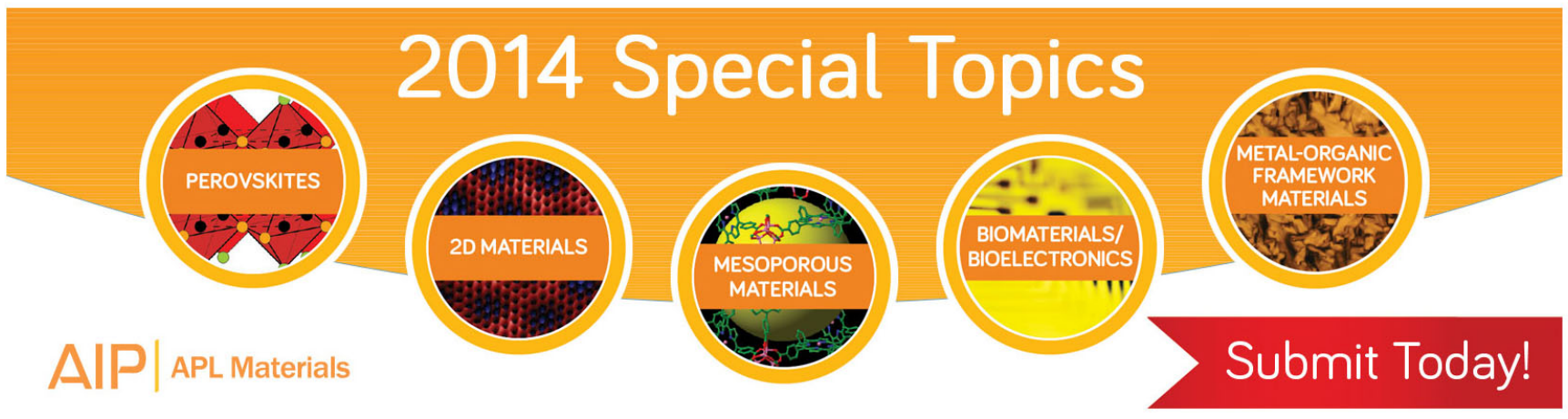




\title{
Ultraviolet laser patterning of porous silicon
}

\author{
Fidel Vega, ${ }^{1, a)}$ Ramón J. Peláez, ${ }^{2}$ Timo Kuhn, ${ }^{2}$ Carmen N. Afonso, ${ }^{2}$ \\ Gonzalo Recio-Sánchez, ${ }^{3, b)}$ and Raúl J. Martín-Palma ${ }^{3}$ \\ ${ }^{1}$ Departament d'Òptica i Optometria, UPC, Violinista Vellsolà 37, 08222 Terrasa, Spain \\ ${ }^{2}$ Laser Processing Group, Instituto de Óptica, CSIC, Serrano 121, 28006 Madrid, Spain \\ ${ }^{3}$ Departamento de Física Aplicada, UAM, Campus de Cantoblanco, 28049 Madrid, Spain
}

(Received 2 April 2014; accepted 25 April 2014; published online 9 May 2014)

\begin{abstract}
This work reports on the fabrication of 1D fringed patterns on nanostructured porous silicon (nanoPS) layers $(563,372$, and $290 \mathrm{~nm}$ thick). The patterns are fabricated by phase-mask laser interference using single pulses of an UV excimer laser $(193 \mathrm{~nm}, 20 \mathrm{~ns}$ pulse duration). The method is a single-step and flexible approach to produce a large variety of patterns formed by alternate regions of almost untransformed nanoPS and regions where its surface has melted and transformed into Si nanoparticles (NPs). The role of laser fluence $\left(5-80 \mathrm{~mJ} \mathrm{~cm}^{-2}\right)$, and pattern period $(6.3-16 \mu \mathrm{m})$ on pattern features and surface structuring are discussed. The results show that the diameter of $\mathrm{Si}$ NPs increases with fluence up to a saturation value of $75 \mathrm{~nm}$ for a fluence $\approx 40 \mathrm{~mJ} \mathrm{~cm}^{-2}$. In addition, the percentage of transformed to non-transformed region normalized to the pattern period follows similar fluence dependence regardless the period and thus becomes an excellent control parameter. This dependence is fitted within a thermal model that allows for predicting the in-depth profile of the pattern. The model assumes that transformation occurs whenever the laser-induced temperature increase reaches the melting temperature of nanoPS that has been found to be 0.7 of that of crystalline silicon for a porosity of around 79\%. The role of thermal gradients across the pattern is discussed in the light of the experimental results and the calculated temperature profiles, and shows that the contribution of lateral thermal flow to melting is not significant for pattern periods $\geq 6.3 \mu \mathrm{m}$. (C) 2014 AIP Publishing LLC. [http://dx.doi.org/10.1063/1.4875378]
\end{abstract}

\section{INTRODUCTION}

Nanostructured porous silicon (nanoPS) has been demonstrated as an excellent candidate for the development of several applications in a broad range of fields including biomedicine, such as drug delivery, tissue engineering, ${ }^{1,2}$ protein immobilization and detection, ${ }^{3,4}$ virus ${ }^{5,6}$ and pesticide ${ }^{7}$ detection, or basic cell studies. ${ }^{8-11}$ The standard method for the fabrication of nanoPS allows tailoring its pore size from the micro- to the nanoscale, as well as its optical properties. Moreover, the optical behavior of nanoPS-based structures $(1 \mathrm{D}, 2 \mathrm{D}$, or $3 \mathrm{D})$ is very sensitive to infiltration ${ }^{12,13}$ or adsorption $^{6}$ of bio-species into the porous material. Furthermore, surface micro- and nano-patterning is becoming an important means for enhancing the performance of materials such as creating superhydrophobic surfaces with hierarchical meshporous structures,${ }^{14}$ reprogramming the cell shape,${ }^{15}$ or enlarging cell culture harvest. ${ }^{16}$ For the particular case of nanoPS, patterns have been used to promote cell binding or growth $^{9-11,17,18}$ or to produce label-free biosensors, ${ }^{3,19}$ the detection mechanism being based on changes either in the photoluminescence spectra or in the diffraction pattern.

Generally, there are two main approaches for fabricating patterns on nanoPS. While the first one consists of microstructuring the crystalline silicon (c-Si) substrate with the desired pattern followed by porosification, ${ }^{17}$ the second and

\footnotetext{
a) Author to whom correspondence should be addressed. Electronic mail: fvega@oo.upc.edu.

b)Present address: Departamento de Ciencias Matemáticas y Físicas, UCT, Campus Norte, Rudecindo Ortega 02950, 4813302 Temuco, Chile.
}

most widely applied method consists of creating the pattern directly on the nanoPS layer by using a diversity of tools such as dry soft lithography, ${ }^{20}$ stamp pressing, ${ }^{19}$ or laser writing. ${ }^{10,21}$ However, none of these methods have the capability to offer flexibility in the pattern design in a timeefficient process, in large areas and in a single-step process. A more versatile approach that has the potential for meeting these requirements is laser interferometry. It was applied in the 1990 s to produce periodic structures in nanoPS ${ }^{22}$ or to take advantage of the photosensitivity of the etching process to produce porous silicon lateral superlattices. ${ }^{23}$ More recently, we have applied it to produce platforms for selective cell culturing. ${ }^{11}$ While the earlier works used two visible laser beams and were focused to morphological aspects rather than to structural properties of the patterns or understanding the underlying mechanism, the recent work used a UV beam in combination with a phase mask and focused on demonstrating that cell selectivity could be achieved through the pattern period. The aim of this paper is to extend this recent work in order to understand the mechanisms controlling pattern formation and eventually determine the possibility of tailoring the aspect ratio of the patterns through the laser fluence.

\section{EXPERIMENTAL}

The nanoPS layers were fabricated by electrochemical etching of boron-doped (p-type) silicon wafers (resistivity: 0.01-0.05 $\Omega \mathrm{cm}$, orientation: $\langle 100\rangle) .{ }^{24}$ The composition of the solution was 1:2 hydrofluoric (HF) (48 wt. \%): ethanol (98 wt. \%). The wafers were galvanostatically etched under 
illumination from a $100 \mathrm{~W}$ halogen lamp. The etching density current was typically $80 \mathrm{~mA} \mathrm{~cm}^{-2}$ and different etching times were used to grow layers of different thickness. The thickness and porosity of the layers have been determined by measuring at both $\mathrm{p}$ and $\mathrm{s}$ polarizations, the spectral reflectance in the $300 \mathrm{~nm}$ to $800 \mathrm{~nm}$ range using a spectroscopic ellipsometer (WVase) at three incidence angles $\left(65^{\circ}, 70^{\circ}\right.$, and $75^{\circ}$ ) and fitting the experimental data using an optical model formed by layers of nanoPS on top of the c-Si substrate. For each layer, an effective medium model which assumes the nanoPS material is a mixture of $\mathrm{Si}$ and air (\% porosity) was used. Aiming at achieving the best possible agreement between the simulated and experimental data, a three layer model was used, consisting in a thin and low porosity bottom layer on top of the c-Si substrate, a thin and intermediate porosity layer, and a thick and high porosity $(74 \%-81 \%)$ top layer. Table I summarizes the thickness and porosity of the layers of the different samples studied in the present work.

In order to fabricate the 1D patterns, single pulses of an excimer laser beam ( $\lambda=193 \mathrm{~nm}, \tau=20 \mathrm{~ns})$ were used to expose a fringed phase mask optimized for high efficiency in the \pm 1 diffraction orders, which were forced to overlap and interfere at the nanoPS surface as described in detail elsewhere. ${ }^{11}$ The nanoPS surface thus becomes exposed to a modulated intensity formed by the maxima and minima of interference. The period of the modulation can easily be modified by using different combinations of projection lenses. In this work, patterns with periods $\Delta$ of $16 \mu \mathrm{m}$, $8.0 \mu \mathrm{m}$, and $6.3 \mu \mathrm{m}$ were studied. While the fluence at the sample site is approximately constant along the fringes $(y$ axis), it is modulated along the direction perpendicular to the fringes ( $x$ axis) according to the following expression:

$$
F(x)=F \cdot(1+\cos (2 \pi x / \Delta))
$$

where $\boldsymbol{F}$ is the average fluence experimentally measured at the sample site that will be referred to from now on as fluence. This means that the actual fluence across the pattern is modulated between 0 and $\boldsymbol{2 F}$. Since an aperture is used to select the central and almost homogeneous part of the laser beam, the smaller the period the broader fluence range achievable. The structural properties of both as-grown and laser processed areas were characterized by field emission scanning electron microscopy (SEM) in planar (PV) and cross-section (CS) views, the latter taken from cleaved samples.

\section{RESULTS}

Figure 1 shows PV (left) and CS (right) SEM images of the nanoPS as-produced samples studied in this work. In PV

TABLE I. Thickness of the 3 nanoPS samples studied in this work as determined from the fit of reflectivity measurements together with the thickness (in $\mathrm{nm}$ ) and porosity $\mathrm{p}$ (in \%) of the 3 layers used in the fit where layer 1 is at the interface with c-Si and layer 3 is the surface layer.

\begin{tabular}{lccc}
\hline \hline Sample thickness & $563 \mathrm{~nm}$ & $372 \mathrm{~nm}$ & $290 \mathrm{~nm}$ \\
\hline Layer 3 & $520 \mathrm{~nm}-74 \%$ & $335 \mathrm{~nm}-81 \%$ & $261 \mathrm{~nm}-79 \%$ \\
Layer 2 & $32 \mathrm{~nm}-65 \%$ & $29 \mathrm{~nm}-70 \%$ & $23 \mathrm{~nm}-63 \%$ \\
Layer 1 & $12 \mathrm{~nm}-54 \%$ & $8 \mathrm{~nm}-57 \%$ & $6 \mathrm{~nm}-55 \%$ \\
\hline \hline
\end{tabular}

images, the surfaces of the nanoPS with an average size pore of $40 \mathrm{~nm}$ are shown. CS images show the longitudinal structure of these pores, and it is worth emphasizing that regardless the nanoPS layer thickness, the c-Si/nanoPS interface has a distinct morphology in comparison to the rest of the nanoPS layer, i.e., it is formed by a mixture of $\mathrm{Si}$ and nanoPS.

The thickness of layer 1 (see Table I) used in the optical simulations is consistent with the thickness of the c$\mathrm{Si} /$ nanoPS interface as measured in the SEM images. In addition, the total thickness of the layers as calculated from the optical simulations (see Table I) agrees (within $<15 \%$ ) with the thickness measured from the CS SEM images

Figure 2 shows both PV and CS images of a $6.3 \mu \mathrm{m}$ period pattern recorded in the $372 \mathrm{~nm}$ thick sample with a fluence of $44 \mathrm{~mJ} \mathrm{~cm}^{-2}$. In the PV image (Figure 2(a)), the 1D pattern is formed by alternate dark and bright areas. The magnified view from Figure 2(b) exhibits the same features than as-grown areas (shown in Figure 1(b)) and evidences that it corresponds to an almost untransformed region, exposed to the laser intensity minima. The magnified view of Figure 2(c), corresponding to the regions exposed to the laser intensity maxima, shows instead a significant transformation with the appearance of several round-like features having diameters of tens of $\mathrm{nm}$ that will be referred to from now on as nanoparticles (NPs). These NPs are on top of a remaining nanoPS layer that is seen in the regions among the NPs.

The low magnification CS image in Figure 2(d) was taken with the sample slightly tilted with respect to the electron beam in order to also visualize the surface. It evidences that the pattern has a topography formed by hills and trenches. Figure 2(e) is made by stitching three consecutive CS-SEM images and shows a magnified view of one of the hills and a portion of the nearby trench. The comparison between the observed features of the hill (structure and thickness) and those of the as-grown sample (Figure 1(b)) evidences that they are almost identical, i.e., the nanoPS layer shows no significant changes in these regions and thus the hills must correspond to the regions exposed to the laser intensity minima similarly to what was deduced from the PV images.

When moving from the hills to the center of the trenches there is a gradual decrease of the nanoPS layer thickness that is accompanied by the formation of the NPs. At the trenches (Figure 2(e)), it is seen that the NPs accumulate but a remnant very shallow nanoPS layer can still be recognized in the CS image. This agrees with the fact that the structure of the nanoPS was observed among the NPs in the PV-SEM image (Figure 2(c)).

Figure 3(a) shows a CS image taken from a trench region of a pattern produced with the same laser fluence $\left(44 \mathrm{~mJ} \mathrm{~cm}^{-2}\right)$ and period $(6.3 \mu \mathrm{m})$ than that shown in Figure 2 but in the thickest layer $(563 \mathrm{~nm})$. By comparing Figure 3 (a) with the one corresponding to the as-grown sample in Figure 1(a), it becomes evident that the NPs produced by the laser are formed on top of a $\approx 390 \mathrm{~nm}$ remnant nanoPS layer and far from the underneath c-Si substrate.

This is in contrast to what was shown in Figure 2(e) for the pattern in the $372 \mathrm{~nm}$ thick sample, in which almost the whole nanoPS layer was converted into NPs at the trenches. 


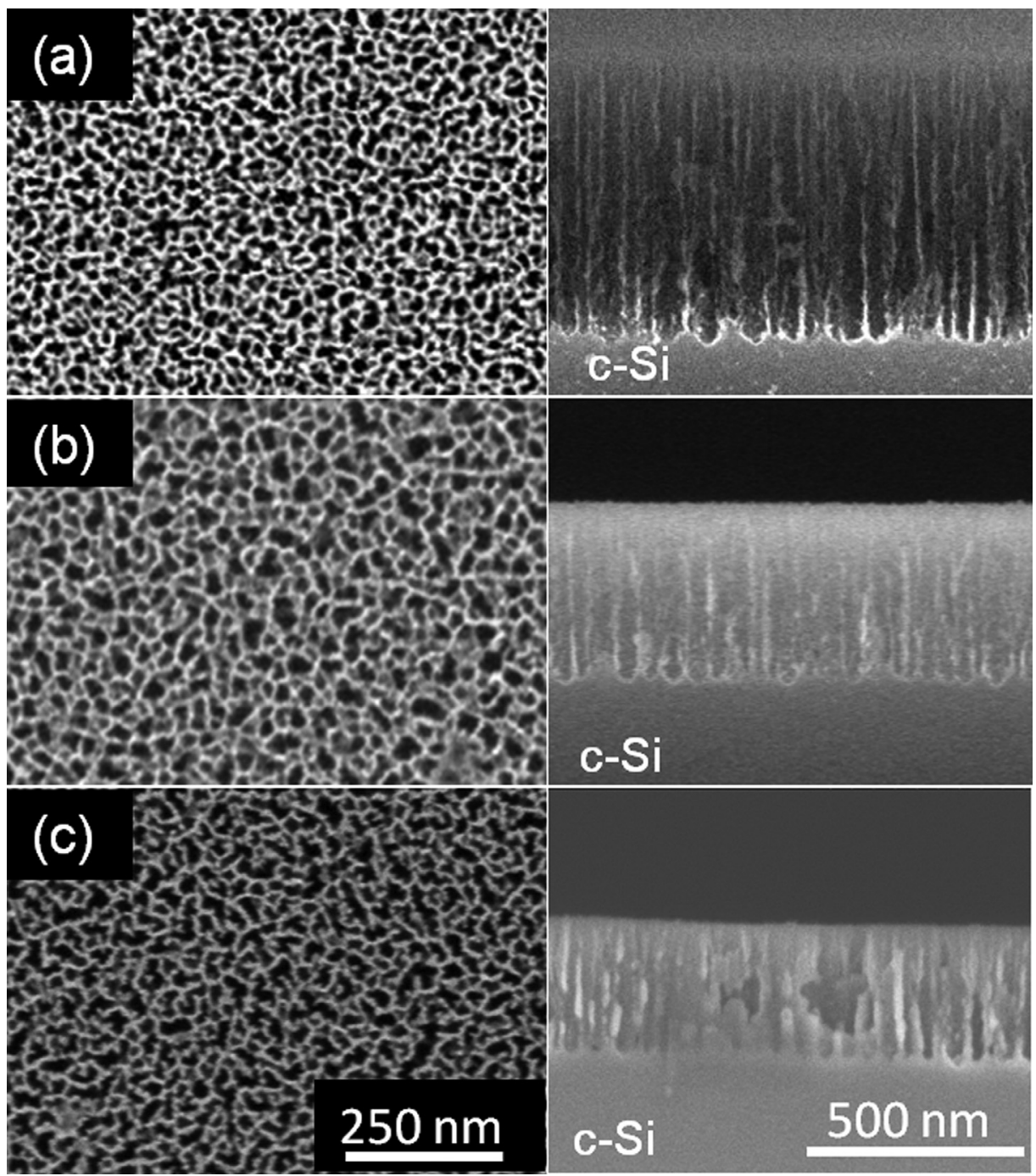

FIG. 1. PV (left) and CS (right) images of the as grown nanoPS layers studied in this work having thicknesses of: (a) $563 \mathrm{~nm}$, (b) $372 \mathrm{~nm}$, and (c) $290 \mathrm{~nm}$.

In order to get further insight on the properties of the NPs, we have immersed this patterned sample in HF solution to dissolve any $\mathrm{SiO}_{2}$ and observed it again in the SEM. The result is shown in Figure 3(b) where the NPs remain
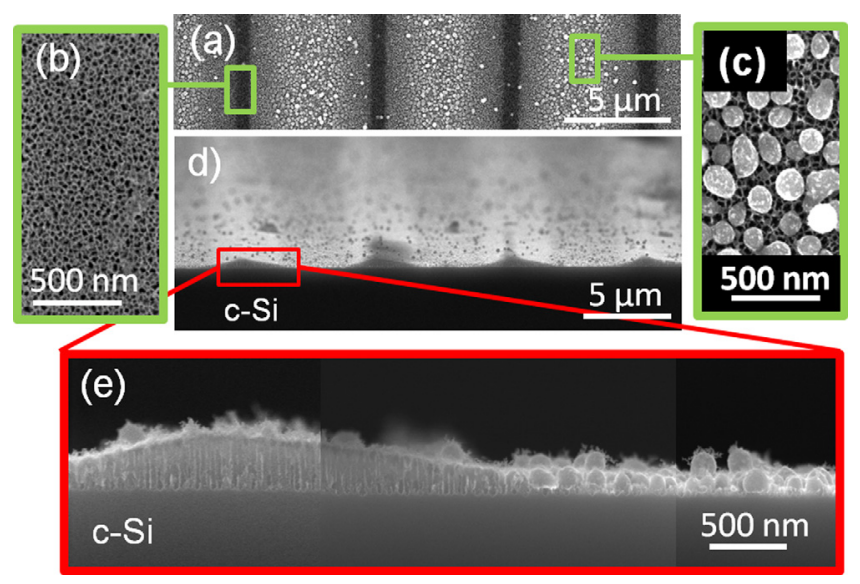

FIG. 2. (a) PV image of a pattern with a period of $6.3 \mu \mathrm{m}$ produced in the $372 \mathrm{~nm}$ thick nanoPS layer using $44 \mathrm{~mJ} \mathrm{~cm}^{-2}$; (b) and (c) are magnification of the dark and bright fringes in (a). (d) Slightly tilted CS image of the same pattern at low magnification. (e) CS image of the same pattern showing, at high magnification, a hill and part of a trench (see text for details). unaffected suggesting that the NPs are formed by silicon and consequently any significant formation of $\mathrm{SiO}_{2}$ in the transformed regions can be discarded. Thus, we will refer from now on to these nanoparticles as Si NPs.

The patterns produced in the $290 \mathrm{~nm}$ thick nanoPS layer with a period of $6.3 \mu \mathrm{m}$ as a function of fluence are shown in Figure 4. Low magnification images of an area exposed to maximum intensity surrounded by areas exposed to minimum intensity and high magnification images of the center of the former areas (i.e., transformed regions) are, respectively, shown on the left and right sides of Figure 4.

From the images on the left, it is seen that the width of the transformed area increases as fluence increases, up to a certain value above which it remains practically constant. From the right side images, it is instead observed that the morphology of the transformed areas are very similar regardless the fluence, i.e., they are covered by Si NPs among which the structure of the nanoPS layer can be seen.

The percentage of transformed to non-transformed region normalized to the pattern period $\Delta$ and determined from images such as those presented in Figure 4, is shown in Figure 5(a) as a function of fluence. A transformation is already observed at low laser fluence $\left(11 \mathrm{~mJ} \mathrm{~cm}^{-2}\right)$ and the fraction of transformed to non-transformed region increases 

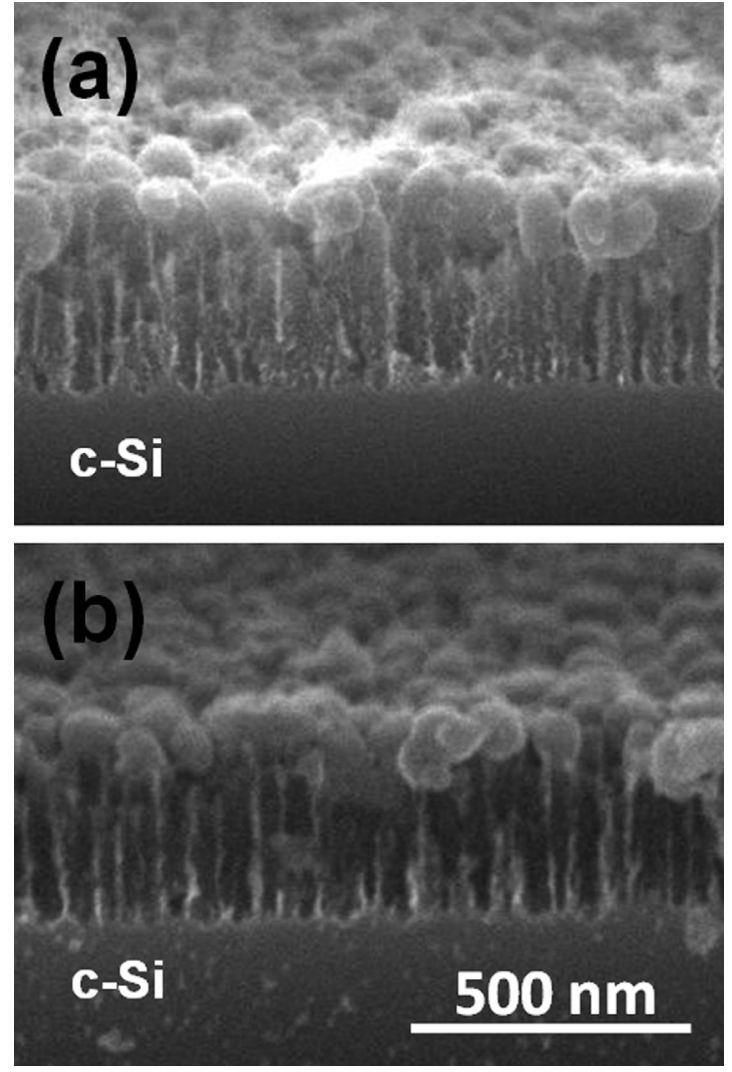

FIG. 3. Slightly tilted CS image of a trench area of a pattern with a period of $6.3 \mu \mathrm{m}$ produced in the $563 \mathrm{~nm}$ thick nanoPS layer using $44 \mathrm{~mJ} \mathrm{~cm}^{-2}$, (a) before and (b) after immersing in HF acid.

first sharply but tends to smoothly saturate for high fluences up to a saturation level of the order of $\approx 0.9 \Delta$. Results achieved for patterns having different periods in a similar sample are also included. It is seen that regardless the period, the behavior is similar and the saturation is reached for a fluence around $40-50 \mathrm{~mJ} \mathrm{~cm}^{-2}$. The diameters of the Si NPs produced in the regions exposed to the intensity maxima as well as their number density have been determined by using the ImageJ software to analyze $1.2 \times 2.0 \mu \mathrm{m}^{2}$ stripes. The results are shown in Figure 5(b) where it is seen that the $\mathrm{Si}$ NPs diameter increases with fluence and also reaches saturation for a fluence $\approx 40 \mathrm{~mJ} \mathrm{~cm}^{-2}$. Figure $5(\mathrm{~b})$ also shows that this increase is mirrored by the number density of these NPs that decreases as fluence increases.

Figure 6 presents CS images of two patterns whose PV images were shown in Fig. 4. Similarly to what was shown in Figure 2(d) for a thicker sample, the untransformed regions correspond to the hills of the topographic profile induced by the laser and have the same thickness than the original nanoPS layer (see Figure 1(c)). Once more, when moving from hills to trenches there is a gradual decrease of the columnar nanoPS layer thickness accompanied by the formation of $\mathrm{Si}$ NPs on top of the remnant underneath nanoPS layer. Increasing fluence leads to a reduction of the width of the hills and, consequently, an increase of the width of the transformed regions. The percentage of transformed to non-transformed region, deduced from these CS images, is also plotted in Figure 5(a) and the values are consistent with the results previously deduced from the PV images.

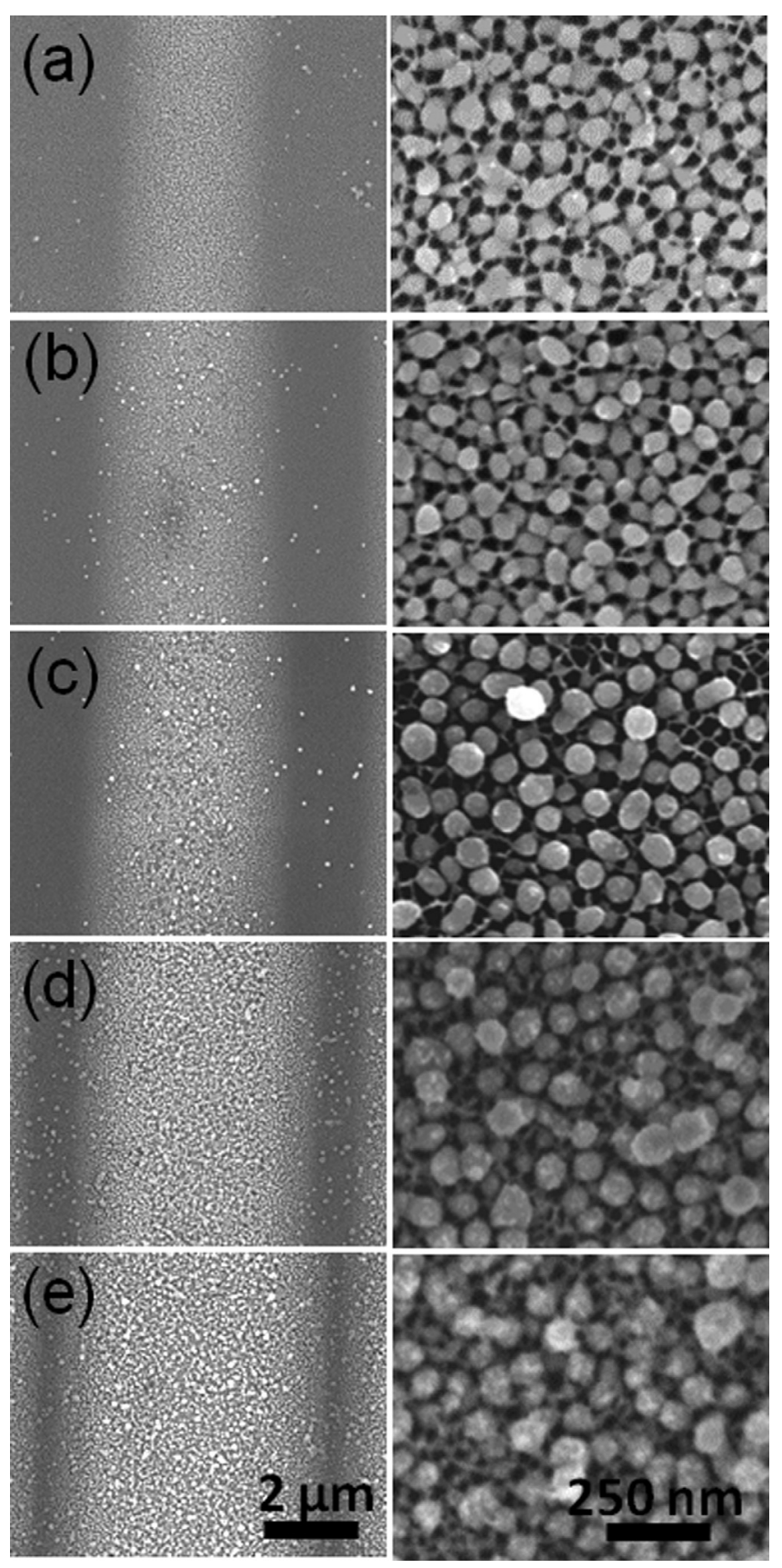

FIG. 4. PV images of patterns with a period of $6.3 \mu \mathrm{m}$ produced in the $290 \mathrm{~nm}$ thick nanoPS layer using increasing fluences in $\mathrm{mJ} \mathrm{cm} \mathrm{cm}^{-2}$ : (a) 11 , (b) 18 , (c) 28 , (d) 42 , and (e) 80 . The images on the left hand side correspond to a low magnification image centered in the trench region whereas the images on the right hand side are high magnifications of the center of the trench regions.

\section{DISCUSSION}

The results plotted in Figure 4 show that alternating transformed and untransformed regions are already produced for a laser fluence of $11 \mathrm{~mJ} \mathrm{~cm}^{-2}$ and $\mathrm{Si}$ NPs are always formed at the transformed areas. The next lower $\mathrm{F}$ studied was $5.5 \mathrm{~mJ} \mathrm{~cm}^{-2}$, which leads to inhomogeneous dark fringes (not shown) with no evidence for NPs formation. Therefore, the transformation threshold will be associated to threshold for NPs formation and must thus be related to laser induced melting. Moreover, the melting fluence $\boldsymbol{F}_{\boldsymbol{m}}$ of the nanoPS layer has to be between $5.5 \mathrm{~mJ} \mathrm{~cm}^{-2}$ and $11 \mathrm{~mJ} \mathrm{~cm}^{-2}$ and more likely closer to the lower value. 


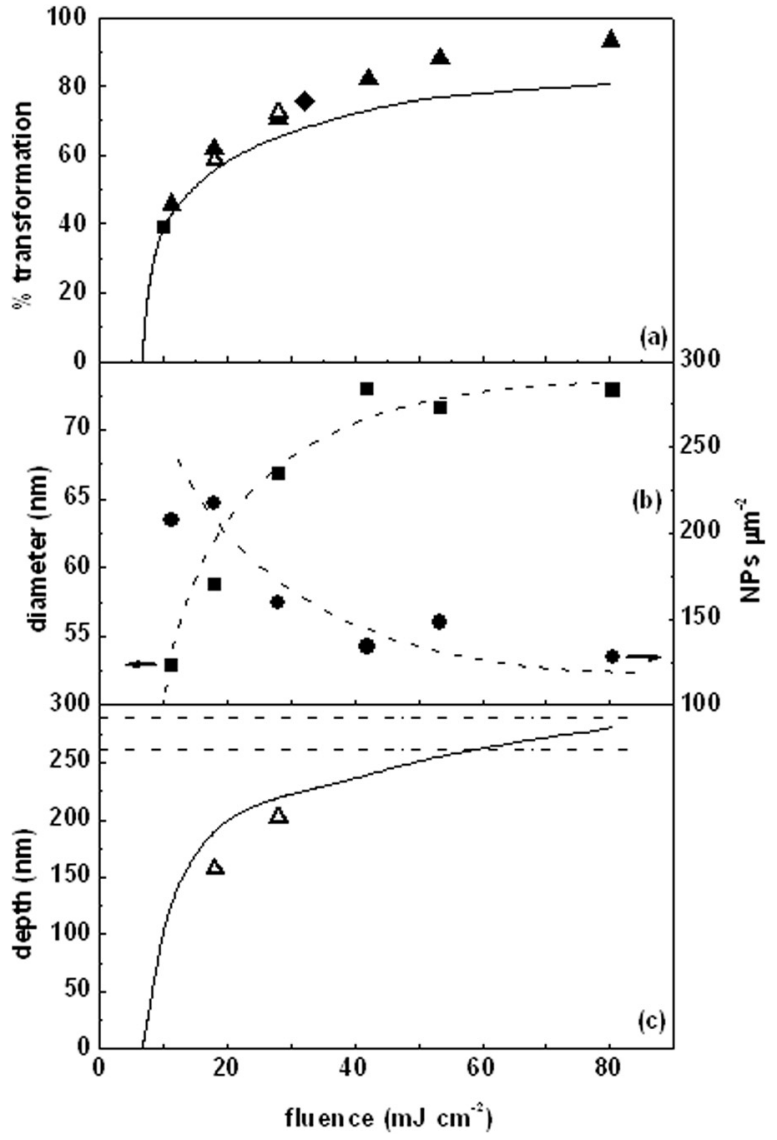

FIG. 5. Several data obtained for the $290 \mathrm{~nm}$ thick sample as a function of fluence: (a) \% of transformed to untransformed regions normalized to the period determined from PV images such as those in Figure 4 from patterns having a period of $(\Delta) 6.3 \mu \mathrm{m},(\diamond) 8 \mu \mathrm{m}$ and $(\mathbf{\square}) 16 \mu \mathrm{m} ;(\triangle)$ data obtained for the $6.3 \mu \mathrm{m}$ period from CS images in Figure 6; (full line)\% calculated using a thermal model. (b) (ם) diameter and (-) number density of Si NPs produced at the trenches of patterns having a period of $6.3 \mu \mathrm{m}$; dashed lines are guidelines. (c) Transformation depth (full line) calculated using a thermal model and $(\triangle)$ experimentally determined form CS images in Figure 6; the horizontal dashed-dot lines represent the total and the upper high porosity layer thickness taken from Table I.

The surface of the nanoPS is exposed to the sinusoidal fluence distribution described by Eq. (1) that eventually leads to alternate regions where the laser fluence is, respectively,

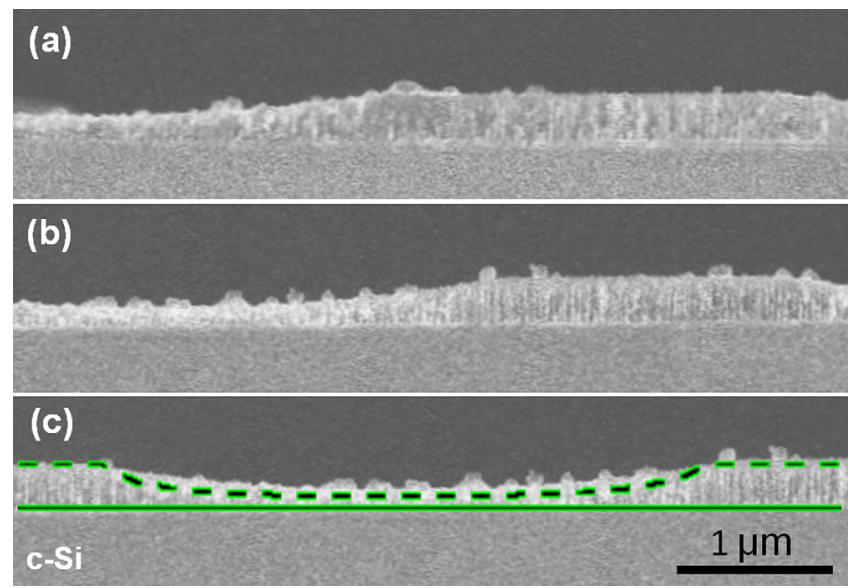

FIG. 6. CS images of patterns with a period of $6.3 \mu \mathrm{m}$ produced in the $290 \mathrm{~nm}$ thick nanoPS layer using fluences in $\mathrm{mJ} \mathrm{cm}^{-2}$ : (a) 18 and (b) 28 . The image in (c) is like (b) but centered at a trench in which the calculated $\boldsymbol{T}_{\boldsymbol{m}}$ isotherm has been overlapped. above and below $\boldsymbol{F}_{\boldsymbol{m}}$. Since the width of the transformed areas should approximately be the extension at which the local fluence is above $\boldsymbol{F}_{\boldsymbol{m}}$, we have fitted the experimentally measured transformed width in Figure 5(a) as a function of $\boldsymbol{F}$ with Eq. (1). A good fit (within 10\%) was achieved and the intersection with the fluence axis provided a value $\boldsymbol{F}_{\boldsymbol{m}}=6.5 \mathrm{~mJ} \mathrm{~cm}^{-2}$ that is very consistent with the experimental predictions of $\boldsymbol{F}_{\boldsymbol{m}}$ being between $5.5 \mathrm{~mJ} \mathrm{~cm}^{-2}$ and $11 \mathrm{~mJ} \mathrm{~cm}^{-2}$. In addition, this value corresponds to an exposure of $2 \boldsymbol{F}_{\boldsymbol{m}}=13 \mathrm{~mJ} \mathrm{~cm}^{-2}$ at the maxima intensity sites. Upon homogeneous beam irradiation at $\lambda=308 \mathrm{~nm}$, a value of $15 \pm 2 \mathrm{~mJ} \mathrm{~cm}^{-2}$ was reported for $\boldsymbol{p}=75 \% .^{25}$ Taking into account on the one hand that the absorption coefficient at $193 \mathrm{~nm}$ is higher than at $308 \mathrm{~nm}$, and on the other hand, that $\boldsymbol{F}_{\boldsymbol{m}}$ decreases as porosity $\boldsymbol{p}$ increases and $\boldsymbol{p}$ is slightly higher in our case (79\%), a lower melting threshold can be expected. Therefore, the $\boldsymbol{F}_{\boldsymbol{m}}$ value determined in this work upon modulated beam exposure agrees with that reported earlier under homogenous beam exposure, ${ }^{25}$ i.e., under conditions for which lateral heat flow (as opposed to in-depth flow) is practically absent. We can therefore conclude that in our case, lateral heat flow from the maxima towards the minima must be also very small, if any, and hence the similar melting threshold found.

In order to get a deeper insight in the melting process, the temperature evolution $\boldsymbol{T}(x, z, t)$ was calculated by solving the heat flow equation for a laser heat source $Q^{26}$

$$
\rho C_{p} \frac{\partial T}{\partial t}=\nabla \cdot(\kappa \cdot \nabla T)+Q
$$

where $\boldsymbol{\rho}$ is the density and $\boldsymbol{\kappa}$ is the thermal conductivity, both parameters depending on the porosity $\boldsymbol{p}$ of the material. The thermal conductivity $\boldsymbol{\kappa}$ of nanoPS as a function of temperature has been obtained from a kinetic model based on the reduction of the effective phonon mean free path where $\boldsymbol{\kappa}$ depends not only on $\boldsymbol{p}$ but also on the center-to-center pore distance ${ }^{27}$ Since according to the reflectivity measurements, the nanoPS layer behaves as a 3-layer system having increased porosity from c-Si up to the surface (see Table I), in Table II the thermal parameters used in the simulations for these three layers as well as for the bulk c-Si underneath have been summarised.

The specific heat capacity $\boldsymbol{C}_{\boldsymbol{p}}$ results to be almost independent of porosity and similar to that of c-Si when applying the equation reported elsewhere for porous silicon. ${ }^{28}$ Therefore, the $\boldsymbol{C}_{\boldsymbol{p}}$ values reported for $\mathrm{c}-\mathrm{Si}$ as a function of temperature have been used. ${ }^{29}$

TABLE II. Parameters used in the temperature calculations for the $290 \mathrm{~nm}$ sample assuming the 3 layer structure showed in Table I for the nanoPS layers. Data for c-Si and liquid Si are also included.

\begin{tabular}{lcccc}
\hline \hline Layer & Thickness $(\mathrm{nm})$ & $P(\%)$ & $\pi\left(\mathrm{kg} \mathrm{m}^{-3}\right)$ & $\kappa @ 300 \mathrm{~K}\left(\mathrm{~W} \mathrm{~m}^{-1} \mathrm{~K}^{-1}\right)$ \\
\hline 3 & 261 & 79 & 490 & 1.25 \\
2 & 23 & 63 & 862 & 3.09 \\
1 & 6 & 55 & 1049 & 4.08 \\
& $\mathrm{c}-\mathrm{Si}$ & 0 & 2330 & 130 \\
& Si (liquid) & 0 & 2570 & 17 \\
\hline \hline
\end{tabular}


To solve Eq. (2), we performed a finite element 3D simulation using the commercial software COMSOL Multiphysics $^{30}$ for the $290 \mathrm{~nm}$ thick sample whose PV and CS images are, respectively, shown in Figures 4 and 6. The heat source corresponds to the fraction of the power density of the laser that is absorbed by the material and is given by ${ }^{26}$

$$
Q(x, z, t)=I(x, t)(1-R) \alpha \exp (-\alpha z),
$$

where in our case, $\boldsymbol{I}(\boldsymbol{x}, \boldsymbol{t})$ is the modulated power density at the surface, $\boldsymbol{R}$ is the reflectivity of the sample at the $\lambda=193 \mathrm{~nm}$ laser wavelength, and $\mathbf{1} / \boldsymbol{\alpha}$ is the optical penetration depth where $\alpha$ is the linear absorption coefficient defined as $\alpha=4 \pi k / \lambda$, and $\boldsymbol{k}$ is the imaginary part of the refractive index. While $\boldsymbol{R}=0.01$ was the value experimentally determined for a close wavelength $(200 \mathrm{~nm}), \boldsymbol{k}=0.39$ was calculated through an effective medium modelling using the optical constants of c-Si and the porosity of the surface layer. This layer is the only one playing a role in the absorption of the laser intensity since the optical penetration depth $(39 \mathrm{~nm})$ results much smaller than the thickness of this layer.

Finally, $I(x, t)$ is determined by both, the spatial and temporal profile of the laser pulse. While the former is described by $\boldsymbol{F}(\boldsymbol{x})$ (Eq. (1)), the latter has to take into account the fact that the excimer laser pulse can be described as the superposition of 3 Gaussian distributions, ${ }^{31}$ with center $\boldsymbol{\mu}_{i}$, relative intensity $\boldsymbol{a}_{i}$ and standard deviation $\boldsymbol{\sigma}_{i}$, leading to

$$
I(x, t)=F(x) \cdot \sum_{i=1}^{3} \frac{a_{i}}{\sigma_{i} \cdot \sqrt{2 \pi}} \exp \left(-\left(t-\mu_{i}\right)^{2} / 2 \cdot \sigma_{i}{ }^{2}\right)
$$

First, the temperature achieved upon irradiation at the calculated $\boldsymbol{F}_{\boldsymbol{m}}=6.5 \mathrm{~mJ} \mathrm{~cm}^{-2}$ value was calculated. It leads to a temperature of $1198 \mathrm{~K}$ at the surface of the sample irradiated with the laser intensity maxima. This temperature is thus considered as the melting temperature $\boldsymbol{T}_{\boldsymbol{m}}$ of our nanoPS material that is significantly smaller than that of c-Si $(1685 \mathrm{~K})^{32}$ consistently with what was earlier reported for nanoPS. ${ }^{25,33}$ In solving Eq. (2), we have considered the material parameters of liquid Si (also included in Table II) for temperatures higher than $\boldsymbol{T}_{\boldsymbol{m}}$. As fluence is increased, the simulations lead to broader regions centered at the intensity maxima sites in which $\boldsymbol{T}_{\boldsymbol{m}}$ is reached at the surface. The calculated width of these regions normalized to the period $\boldsymbol{\Delta}$ is also plotted in Figure 5(a) as a function of fluence where they follow the same trend than the experimental values. Moreover, the calculations also show a tendency to saturate to a value $\approx 0.8 \Delta$ that is lower than the experimental one. The existence of a saturation in both experimental and calculated values means that there is always a non-transformed region (around the intensity minima sites) for the studied range of periods. Since the laser fluence at the minima of interference is zero, the only way this region could be heated would be through lateral heat flow from hotter adjacent regions. However, the calculations show that for the highest fluence, $\left(80 \mathrm{~mJ} \mathrm{~cm}^{-2}\right)$ the temperature of the regions around the intensity minima are at the most $0.4 \boldsymbol{T}_{\boldsymbol{m}}$. Furthermore, this temperature represents an upper limit since real values are expected to be smaller because the thermal conductivity of nanoPS in the direction perpendicular to the pores have been reported to be smaller than along the pores. ${ }^{34}$ It is worth to point out here that the described scenario is valid for the range of periods studied in this work $(\geq 6.3 \mu \mathrm{m})$ and might change if the period is significantly reduced.

The in-depth $\boldsymbol{T}_{\boldsymbol{m}}$ isotherm calculated for a period of $6.3 \mu \mathrm{m}$ and $28 \mathrm{~mJ} \mathrm{~cm}^{-2}$ once the maximum depth and width are achieved, is overlapped to the experimental CS image in Figure 6(c). It is seen that the calculated thermal profile matches well the experimental shape and depth of the trench and supports further that melting is nucleated around the sites exposed to the maxima of interference and propagates in-depth. We have defined the melting depth as the maximum depth of these $\boldsymbol{T}_{\boldsymbol{m}}$ isotherms and the results as a function of fluence are plotted in Figure 5(c). It can be observed that the trend of this depth is very similar to that of the relative transformation width deduced from PV images and plot in Figure 5(a), i.e., it increases first sharply and tends to saturate for high fluences. Furthermore, melting has affected the whole upper layer of high porosity of the sample for fluences around $60 \mathrm{~mJ} \mathrm{~cm}^{-2}$. The experimental depth of transformation is obtained from the CS-SEM images in Figure 6 as the height difference between trenches and hills and the results are also included in Figure 5(c). The good agreement between the calculated melting depths and experimental transformation depths confirms further that transformation of nanoPS into Si NPs is a consequence of in-depth melting.

Additionally, our results show that even for the higher fluences for which the original nanoPS layer would be expected to have completely molten and converted into $\mathrm{Si}$ NPs, the porous features of the as-grown layer are always recognized among the NPs (Figure 4). In other words, a remnant nanoPS layer must remain at the c-Si interface (Figures 2(e) and 4). The difficulty to melt completely and transform this interface was also suggested earlier ${ }^{33}$ and is most likely related to the high efficiency of the c-Si as a heat sink since its thermal conductivity is one or two order of magnitude higher than that of nanoPS. Therefore, the closer the melt front to the nanoPS/c-Si interface, the higher the thermal gradient as the heat is efficiently drained out through the interface to the c-Si substrate. This efficient heat sink together with the higher melting temperature of the nanoPS region close to the c-Si interface due to its lower porosity contribute to prevent reaching the melting temperature. This reasoning is consistent with the saturation observed in Figures 4, 5(a), and 5(c) for the width and depth of the trenches as fluence is increased.

The formation of Si NPs has earlier been reported under homogeneous beam irradiation and similarly related to melting. ${ }^{25,33}$ In our case, the NPs are either on top of a large remnant columnar structure of nanoPS and thus far from the c-Si substrate for the thickest layer studied (Figure 3), or they are on top of a shallow remnant nanoPS layer that is close to the interface with the c-Si substrate (for the higher fluences and the lower layer thicknesses studied). The fact that NPs rather than a continuous layer are formed upon melting is most likely related to the rough and discontinuous character of the nanoPS surface that induces heterogeneously nucleated melting at discrete points of the surface as suggested elsewhere. ${ }^{25}$ 
This leads to the formation of small "islands" of molten material that evolve to NPs promoted by the higher surface energy of molten Si than that of the remnant nanoPS. Figure 5(b) shows that the diameter of the NPs increases in the range of $50-73 \mathrm{~nm}$ as fluence is increased. This increase is consistent with the increase of thickness of the molten nanoPS layer as fluence increases. However, Fig. 5(b) also shows that the number density of NPs decreases as their diameter increases thus suggesting that the diameter increase is related to both the increase of the transformation depth and to aggregation of nearby NPs.

Finally, the results presented here show that for a given layer thickness and pattern period, the width and depth of the fringes of the patterns as well as the dimensions of the $\mathrm{Si}$ NPs formed at the trenches at the expense of the nanoPS layer can be controlled by the fluence. The possibility of tuning the topography as well as the physical and chemical properties of the surface through the conversion of nanoPS into Si NPs is essential for cell culturing since it was shown that cells bound and align to the patterns whenever the width of the trenches were large enough to accommodate the cells. ${ }^{11}$ It was suggested that the laser induced trenches act as microfluidic wells for the cells during their colonization of the surface. Moreover, the fact that the cells bound preferentially to the trenches most likely indicated that the physical and chemical properties at these sites improve the wetability and adhesive properties for cells. ${ }^{35}$

\section{CONCLUSIONS}

Single pulse UV laser interference has been demonstrated as a flexible tool to fabricate 1D topographic patterns in nanoPS with several periods and having a wide range of aspect ratios. These patterns are formed by regions of hills and trenches, each related to almost untransformed regions having similar thickness and structure than the nanoPS original layer and regions where the nanoPS structure has melted and converted into Si NPs. Within the range of pattern periods and fluences studied, these features are found to be almost independent of period for periods $\geq 6.3 \mu \mathrm{m}$. The aspect ratio of the patterns as well as the diameter of the $\mathrm{Si}$ NPs produced at the trenches can easily be tailored through the laser fluence.

The melting threshold of nanoPS upon modulated beam exposure has been experimentally determined, and the value agrees with that reported earlier upon melting using a homogeneous beam exposure. The calculations made with a standard thermal model reproduce the fluence dependence of the relative width and depth of trenches as well as their shape and allow concluding that transformation occurs in regions that reach melting temperature. A melting temperature 0.7 times smaller than that of $\mathrm{c}-\mathrm{Si}$ was determined and this value is consistent with what has earlier been reported for nanoPS layers of similar porosity. The saturation observed in both the experimental and calculated relative width and depth of the transformed regions as fluence increases is consistent with the higher melting threshold of the nanoPS layer in the neighborhood of the interface with c-Si together with the much higher thermal conductivity of the latter. All together, the results allow also concluding that thermal gradients across the pattern promote no significant lateral temperature increase and the achieved temperature in the regions irradiated with the minima of intensity is far from the melting temperature in the studied range of fluences and layer thicknesses.

\section{ACKNOWLEDGMENTS}

This work has partially been supported under projects MAT2011-28345-C02-01 and MAT2011-28345-C02-02 (Spain). R. J. Peláez acknowledges the grant JCI2012_13034 from the Juan de la Cierva program. T. Kuhn acknowledges the support of DAAD. The authors gratefully thank L. García Pelayo for technical support and the Electron Microscopy Laboratory of CENIM (CSIC) for helping with the microscope.

${ }^{1}$ E. J. Anglin, L. Cheng, W. R. Freeman, and M. J. Sailor, Adv. Drug Deliv. Rev. 60, 1266 (2008).

${ }^{2}$ R. J. Martín-Palma, M. Manso-Silván, and V. Torres-Costa, J. Nanophoton. 4, 042502 (2010).

${ }^{3}$ I. Kleps, M. Miu, M. Simion, T. Ignat, A. Bragaru, F. Craciunoiu, and M. Danila, J. Biomed. Nanotechnol. 5, 300 (2009).

${ }^{4}$ L. De Stefano, L. Rotiroti, I. Rendina, L. Moretti, V. Scognamiglio, M. Rossi, and S. D'Auria, Biosens. Bioelectron. 21, 1664 (2006).

${ }^{5}$ A. M. Rossi, L. Wang, V. Reipa, and T. E. Murphy, Biosens. Bioelectron. 23, 741 (2007).

${ }^{6}$ Y. Vashpanov, J. Son, K. Kwack, and S. Shing, Jpn. J. Appl. Phys., Part 1 47, 5100 (2008)

${ }^{7}$ L. De Stefano, L. Moretti, I. Rendina, and L. Rotiroti, Sens. Actuators, B 111-112, 522 (2005).

${ }^{8}$ A. Angelescu, I. Kleps, M. Mihaela, M. Simion, T. Neghina, S. Petrescu, N. Moldovan, C. Paduraru, and A. Raducanu, Rev. Adv. Mater. Sci. 5, 440 (2003); available at http://www.ipme.ru/e-journals/RAMS/index.html.

${ }^{9}$ A. D. Doyle, F. W. Wang, K. Matsumoto, and K. M. Yamada, J. Cell Biol. 184, 481 (2009).

${ }^{10}$ Y.-L. Khung, S. D. Graney, and N. H. Voelcker, Biotechnol. Prog. 22, 1388 (2006).

${ }^{11}$ R. J. Peláez, C. N. Afonso, F. Vega, G. Recio-Sánchez, V. Torres-Costa, M. Manso-Silván, J. P. García-Ruiz, and R. J. Martín-Palma, J. Biomed. Mater. Res. Part B 101B, 1463 (2013).

${ }^{12}$ J. J. Saarinem, S. M. Weiss, P. M. Fauchet, and J. E. Sipe, Opt. Express 13, 3754 (2005).

${ }^{13}$ R. J. Martín-Palma, M. Manso, and V. Torres-Costa, Sensors 9, 5149 (2009).

${ }^{14}$ J. Yong, Q. Yang, F. Chen, D. Zhang, H. Bian, Y. Ou, J. Si, G. Du, and X. Hou, Appl. Phys. A 111, 243 (2013).

${ }^{15}$ T. Vignaud, R. Galland, Q. Tseng, L. Blanchoin, J. Colombelli, and M. Théry, J. Cell Sci. 125, 2134 (2012).

${ }^{16}$ M. Hoffmann, J.-P. Kuska, M. Zscharnack, M. Loeffler, and J. Galle, PLoS One 6, e21960 (2011).

${ }^{17}$ V. Torres-Costa, G. Martínez-Muñoz, V. Sánchez-Vaquero, Á. MuñozNoval, L. González-Méndez, E. Punzón-Quijorna, D. Gallach-Pérez, M. Manso-Silván, A. Climent-Font, J. P. García-Ruiz, and R. J. MartínPalma, Int. J. Nanomed. 7, 623 (2012).

${ }^{18}$ Á. Muñoz-Noval, V. Sánchez-Vaquero, E. Punzón-Quijorna, V. TorresCosta, D. Gallach-Pérez, L. González-Méndez, I. Montero, R. J. MartínPalma, A. Climent-Font, J. P. García-Ruiz, and M. Manso-Silván, J. Biomed. Mater. Res. Part A 100A, 1615 (2012).

${ }^{19}$ J. D. Ryckman, M. Liscidini, J. E. Sipe, and S. M. Weiss, Appl. Phys. Lett. 96, 171103 (2010).

${ }^{20}$ B. D. J. Sirbuly, G. M. Lowman, B. Scott, G. D. Stucky, and S. K. Buratto, Adv. Mater. 15, 149 (2003).

${ }^{21}$ L. De Stefano, I. Rea, M. Arcangela-Nigro, F. G. Della Corte, and I. Rendina, J. Phys.: Condens. Matter 20, 265009 (2008).

${ }^{22}$ V. I. Vlad, A. Petris, V. N. Chumash, and I. Cojocaru, Appl. Surf. Sci. 106, 356 (1996). 
${ }^{23}$ G. Lérondel, R. Romestain, J. C. Vial, and M. Thoünissen, Appl. Phys. Lett. 71, 196 (1997).

${ }^{24}$ L. T. Canham, Appl. Phys. Lett. 57, 1046 (1990).

${ }^{25}$ V. Y. Timoshenko, T. Dittrich, I. Sieber, J. Rappich, B. V. Kamenev, and P. K. Kashkarov, Phys. Status Solidi 182, 325 (2000).

${ }^{26} \mathrm{M}$. von Allmen, Laser Beam Interactions with Materials, Materials Science Vol. 2 (Springer, Berlin, 1987),.

${ }^{27}$ J. Boor, D. S. Kim, X. Ao, D. Hagen, A. Cojocaru, H. Föll and V. Schmidt, EPL 96, 16001 (2011).

${ }^{28}$ E. Amin-Chalhoub, N. Semmar, L. Coudron, G. Gautier, C. BoulmerLeborgne, A. Petit, M. Gaillard, J. Mathias, and E. Millon, J. Phys. D: Appl. Phys. 44, 355401 (2011).
${ }^{29}$ C. P. Grigoropoulos, H. K. Park, and X. Xu, Int. J. Heat Mass Transfer 36, 919 (1993).

${ }^{30}$ See www.comsol.com for COMSOL Multiphysics 4.3 Documentation.

${ }^{31}$ F. Vega, C. N. Afonso, W. Szyszko, and J. Solis, J. Appl. Phys. 82, 2247 (1997).

${ }^{32}$ B. Rezek, C. E. Nebel, and M. Stutzmann, J. Appl. Phys. 91, 4220 (2002).

${ }^{33}$ T. Dittrich, I. Sieber, W. Henrion, S. Rauseher, N. Wanderka, and J. Rappich, Appl. Phys. A Mater. Sci. Process. 63, 467 (1996).

${ }^{34}$ J. D. Chung, KSME Int. J. 13, 762 (1999).

${ }^{35}$ C. C. Berry, G. Campbell, A. Spadiccino, M. Robertson, and A. S. G. Curtis, Biomaterials 25, 5781 (2004). 